\title{
Direct searches for the standard model Higgs boson produced in association with a vector boson at CDF
}

\author{
Weiming Yao*t \\ Lawrence Berkeley National Lab \\ E-mail: wmyao@lbl.gov
}

\begin{abstract}
We present the results of searches for the standard model Higgs boson at CDF in final states with bottom quarks. Results are derived from the complete Tevatron Run II dataset, with a measured integrated luminosity of $9.5 \mathrm{fb}^{-1}$ of proton-antiproton data. The searches are performed for assumed Higgs masses between 90 and $150 \mathrm{GeV}$, for Higgs bosons produced in association with $\mathrm{W}$ or $\mathrm{Z}$ bosons. Employing several improved techniques, these are currently the most sensitive searches in the world for these processes, surpassing previous CDF results by $30 \%$ beyond what would be expected from the addition of new data alone. Combining the search sensitivity of these production modes, $95 \%$ upper confidence limits on the standard model cross section times branching fraction are derived, yielding an observed (expected) upper limit of 4.3 (1.8) times the standard model prediction for a $125 \mathrm{GeV}$ Higgs boson. The significance of the data relative to the background-only hypothesis is 2.5 sigma.
\end{abstract}

36th International Conference on High Energy Physics,

July 4-11, 2012

Melbourne, Australia

\footnotetext{
*Speaker.

†n behalf of the CDF Collaboration.
} 


\section{Introduction}

Understanding the mechanism for electroweak symmetry breaking, especially by testing for the presence or absence of the standard model (SM) Higgs boson [1], has been a major goal of particle physics and a central part of the Fermilab Tevatron physics program. Both CDF and D0 collaborations have performed direct searches for the standard model Higgs boson [2]. The new searches reported here include more data, additional channels, and improved analyses techniques compared to previous analyses. Results are derived from the complete Tevatron Run II dataset, with a measured integrated luminosity of $9.5 \mathrm{fb}^{-1}$ of proton-antiproton data. The searches are performed for assumed Higgs masses between 90 and $150 \mathrm{GeV} / \mathrm{c}^{2}$.

The SM does not predict the mass of Higgs boson, $m_{H}$, but the global fit of the electroweak precision data, including recent top-quark and $W$ boson mass measurements from the Tevatron [3, 4], constrains $m_{H}$ to be less than $152 \mathrm{GeV} / \mathrm{c}^{2}$ at the $95 \%$ conference level (CL) [5]. The direct searches from LEP [6], Tevatron [2], and LHC results [7, 8] set the Higgs mass between 116.6 and 119.4 $\mathrm{GeV} / \mathrm{c}^{2}$ or between 122.1 and $127 \mathrm{GeV} / \mathrm{c}^{2}$ at the $95 \% \mathrm{CL}$. Recently both LHC experiments $[9,10]$ observed local excesses above the background expectations for a Higgs boson mass of approximately $125 \mathrm{GeV} / \mathrm{c}^{2}$. Much of the power of the LHC searches comes from $g g \rightarrow H$ production and Higgs boson decays to $\gamma \gamma, W^{+} W^{-}$, and $Z^{+} Z^{-}$, which probe the couplings of the Higgs boson to other bosons. In the allowed mass range, the Tevatron experiments are particularly sensitive to the associated production of the Higgs boson with a weak vector boson in the $b \bar{b}$ channel, which probes the coupling of the Higgs boson to $b$ quarks.

The Tevatron collider produces proton and anti-proton collision at the center mass of $1.96 \mathrm{TeV}$ with a record luminosity of $4.310^{32} \mathrm{~cm}^{-2} \mathrm{~s}^{-1}$. The Tevatron delivered close to $12 \mathrm{fb}^{-1}$ to each experiment before the shutdown on 30 September 2011. The CDF detector is a general-purpose detector, which provides excellent tracking, lepton identification, jets finding, missing transverse energy ( $\mathbb{E}_{T}$ ) detection, and efficient multilevel triggers. The details can be found elsewhere [11].

\section{Search Strategies}

The dominant Higgs production processes at the Tevatron are the gluon-gluon fusion ( $g g \rightarrow H$ ) and the associated production with a $W$ or $Z$ boson [12]. The cross section for $W H$ production is twice that of $Z H$ and is about a factor of 10 smaller than $g g \rightarrow H$. The Higgs boson decay branching fraction is dominated by $H \rightarrow b \bar{b}$ for the low-mass Higgs $\left(m_{H}<135 \mathrm{GeV} / \mathrm{c}^{2}\right)$ and by $H \rightarrow W^{+} W^{-}$ or $Z Z^{*}$ for the high-mass Higgs $\left(m_{H}>135 \mathrm{GeV} / \mathrm{c}^{2}\right)$. A search for a low-mass Higgs boson in the $g g \rightarrow H \rightarrow b \bar{b}$ channel is extremely challenging because the $b \bar{b}$ QCD production rate is many orders of magnitude larger than the Higgs boson production rate. Requiring the leptonic decay of the associated $W$ or $Z$ boson greatly improves the expected signal over background ratio in these channels. As a result, the Higgs associated production with $H \rightarrow b \bar{b}$ is the most promising channel for the low-mass Higgs boson searches at the Tevatron.

The search strategies employed at CDF have been evolving constantly over time. We first maximize the signal acceptance by using efficient triggers, excellent lepton identifications, and powerful $b$-tagging, which can improve the signal to background ratio up to the $1 \%$ level. Then we use multivariate analysis (MVA) to exploit the kinematic differences between signal and back- 
ground, which can further enhance the signal to background ratio up to the $10 \%$ level in the high score regions. The same strategies have been used to help discover the single-top and diboson processes at the Tevatron, and provide a solid ground for isolating a small signal out of a large background.

For the $H \rightarrow b \bar{b}$ signatures we look for a $b \bar{b}$ mass resonance produced in association with a $W$ or $Z$ boson where $W$ decays into $l v$ or $Z$ decays into $l^{+} l^{-}$or $v \bar{v}$. The $W H \rightarrow l v b b$ is the most sensitive channel that gives one high $P_{T}$ lepton, large $\mathbb{E}_{T}$, and two $b$-jets. Before $b$-tagging, the sample is predominated by the $W+$ light-flavor jets, which provides an ideal control data to test the background modeling.

Since there are two $b$-quark jets from the low-mass Higgs decay, improving $b$-tagging is crucial. CDF use multivariate $b$-taggers to exploit the decay of long-lived $B$ hadron as displaced tracks/vertices. The typical $b$-tag efficiency is about $40-70 \%$ with a mistag rate of $1-5 \%$ per jet. Recently CDF combined their existing $b$-taggers into a Higgs Optimized $b$-tagger (HOBIT) [13] using a neural network tagging algorithm, based on sets of kinematic variables sensitive to displaced decay vertices and tracks within jets with large transverse impact parameters relative to the hard-scatter vertices. Using an operating point which gives an equivalent rate of false tags, the new algorithm improves upon previous $b$-tagging efficiencies by $\approx 20 \%$.

\section{3. $H \rightarrow b \bar{b}$ Searches}

We describe the searches for the low-mass Higgs boson with $H \rightarrow b \bar{b}$ at CDF.

\section{1 $W H \rightarrow l v b \bar{b}$}

One of the golden channels for the low-mass Higgs boson search at the Tevatron is the Higgs produced in association with a $W$ boson with $W H \rightarrow l v b \bar{b}$ [14]. We select events with one isolated high $P_{T}$ lepton (electron, muon, or isolated track), a large missing transverse energy, and two or three jets, of which at least one is required to be $b$-tagged as containing a weakly-decaying $\mathrm{B}$ hadron. Events with more than one isolated lepton are rejected. For the multivariate discriminant, we train a Bayesian neural network discriminant $(\mathrm{BNN})$ in the $W+$ two or three jets for each Higgs mass, separately for each lepton type, jet multiplicity, $b$-tagging category.

We perform a direct search for an excess of events in the signal region of the final discriminant from each event category. Since there is no significant excess of signal events observed in the data, we set an upper limit at 95\% CL on the Higgs production cross section times branching ratio with respect to the SM predictions as a function of Higgs mass. CDF set an observed (expected) upper limit at 4.9(2.8) for $m_{H}=125 \mathrm{GeV} / \mathrm{c}^{2}$.

\section{2 $\mathrm{ZH} \rightarrow l^{+} l^{-} b \bar{b}$}

Another interesting channel to search for the low-mass Higgs boson is $Z H \rightarrow l^{+} l^{-} b \bar{b}$ [15]. It has a clean signature, but a low event yield due to a small branching fraction of $Z \rightarrow e^{+} e^{-}$and $\mu^{+} \mu^{-}$. We select events with two high $P_{T}$ leptons from $Z$ decay and two or three jets. Events are further divided based on lepton type, jet multiplicity, and the number of $b$-tagged jets, similar to $W H \rightarrow l v b \bar{b}$. To increase signal acceptance we use neural networks to select loose dielectron and dimuon candidates. The jet energies are corrected for the missing $E_{T}$ using a neural network 
approach. We utilize a multi-layer discriminant based on neural networks where separate discriminant functions are used to define four separate regions of the final discriminant function. There seems to be an excess of events in the high score signal region, but nothing statistically significant yet. We set an observed (expected) upper limit at 95\% CL on the Higgs cross section times branching ratio over the standard model prediction at 7.2 (3.6) for the Higgs mass at $125 \mathrm{GeV} / \mathrm{c}^{2}$.

\section{3 $W H, Z H \rightarrow \mathbb{E}_{T} b \bar{b}$}

We also search for the Higgs boson in the $Z H$ and $W H$ channels where the $Z$ boson decays into two neutrinos or the lepton from the $W$ decay is undetected [16]. It has a large signal rate as well as a large QCD-multijet background. However, the final state is relatively clean, containing two high $E_{T}$ jets and a large missing transverse energy. We require $\mathbb{E}_{T}>50 \mathrm{GeV}$ and two $b$-tagged jets. We use a track-based $\mathbb{E}_{T}$ calculation as a discriminant against false $\mathbb{E}_{T}$. In addition we utilize a neural network to further discriminate against the multi-jet background. The final discriminant is obtained for a Higgs signal by combining dijet mass, track $\mathbb{E}_{T}$, and other kinematic variables. The data are consistent with the background expectations; we set an observed (expected) upper limit at 95\% CL on the Higgs cross section times branching ratio over the standard model prediction at 6.8 (3.6) for the Higgs mass at $125 \mathrm{GeV} / \mathrm{c}^{2}$.

\section{Combination of $H \rightarrow b \bar{b}$}

We combine searches for $H \rightarrow b \bar{b}$ in three most promising channels, and set limits with respect to nominal SM predictions [17]. We combine the results using a combined likelihood formed from a product of likelihoods for the individual channels. Systematic uncertainties are treated as nuisance parameters with truncated Gaussian. The common systematics between channels are the luminosity, detector related efficiencies, theoretical cross sections, and PDF uncertainties, which are treated as correlated. The instrumental backgrounds are treated as independent. Most of nuisance parameters are well constrained by the data in the dominant background region and are not very sensitive to the initial-input values.

To validate our background modeling and search methods, we additionally perform a search for $Z \rightarrow b \bar{b}$ in association with a $W$ or $Z$ boson using the same final states of the SM $H \rightarrow b \bar{b}$ searches. The data sample, reconstruction, background models, uncertainties, and sub-channel divisions are identical to those of the SM Higgs boson search, but the discriminant functions are trained using the signal of $Z \rightarrow b \bar{b}$ instead. The measured cross section of $W Z+Z Z$ is $4.1 \pm 1.3$ $\mathrm{pb}$, which is consistent with the SM prediction of $4.4 \pm 0.3 \mathrm{pb}$.

To further check the consistency between data and background, we rebin the final discriminant from each channel in terms of the signal to background ratio $(\mathrm{s} / \mathrm{b})$ so that the data with similar $\mathrm{s} / \mathrm{b}$ may be added without loss of sensitivity. The resulting data distributions after background subtraction are shown in figure 1 (left) and compared to the expected signal of $m_{H}=125$. There seems to be a small excess of Higgs boson candidate events in the highest s/b bins relative to the background-only expectations.

Figure 1 (right) shows the combined limit of $H \rightarrow b \bar{b}$ after combining three channels together. We start to exclude the Higgs mass near the low-mass end between $90<m_{H}<96 \mathrm{GeV} / \mathrm{c}^{2}$ and obtain an observed (expected) limit of 4.15 (1.8) at 95\% CL on the SM Higgs production cross 

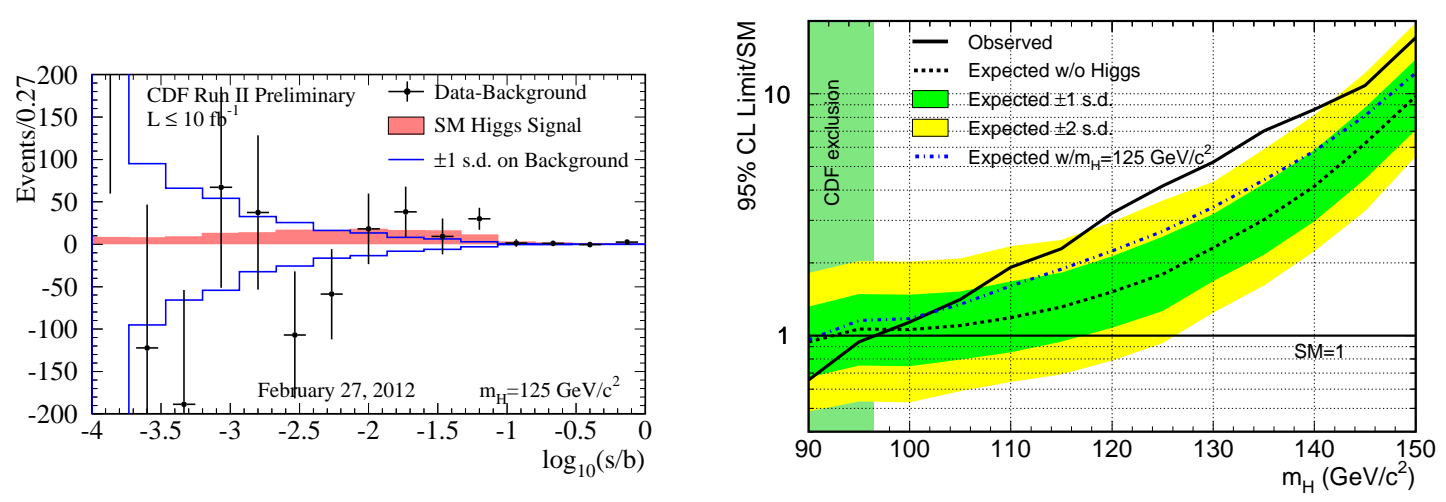

Figure 1: (Left) Background-subtracted data distributions for the discriminant histograms, summed for bins with similar s/b, for $m_{H}=125 \mathrm{GeV} / \mathrm{c}^{2}$. (Right) Observed and expected 95\% CL upper limits on SM Higgs boson production cross section with respect to SM predictions as a function of $m_{H}$. The shaded bands indicate the one or two sigma regions where the limit is expected to fluctuate, in the absence of signal. The dot-dashes are the expected limits with the SM Higgs signal of $m_{H}=125 \mathrm{GeV} / \mathrm{c}^{2}$.

section with respect to the SM prediction for $m_{H}=125 \mathrm{GeV} / \mathrm{c}^{2}$. There seems to be a small excess of events between 115 and $140 \mathrm{GeV} / \mathrm{c}^{2}$. We quantify the excess by calculating the local $\mathrm{p}$-value for the background-only hypothesis. Figure 2 (left) shows the local p-value as a function of Higgs mass where the solid curve is for data and the dashed curve is what is expected from the SM Higgs production of $m_{H}=125 \mathrm{GeV} / \mathrm{c}^{2}$. At $m_{H}=135 \mathrm{GeV} / \mathrm{c}^{2}$, we have a minimum local $\mathrm{p}$-value $=0.32 \%$ or 2.7 sigma, which corresponds to a global p-value $=2.5$ sigma including a LEE factor of 2 . Given the excess we also fit the signal cross section times the decay branching ration as a function of Higgs mass as shown in Figure 2 (right). We measure an associated production cross section times the decay branching ratio of $\left(\sigma_{W H}+\sigma_{Z H}\right) \times B(H \rightarrow b \bar{b})=291_{-113}^{+118}$ (stat+sys) fb, which is consistent with the SM prediction of $120 \pm 10 \mathrm{fb}$ for $m_{H}=125 \mathrm{GeV} / \mathrm{c}^{2}$.

\section{Conclusion}

In conclusion, with a full dataset and many years of hard work, the CDF Collaboration has finally exceeded their most optimistic sensitivity projection based on 2007 summer results. The searches are conducted for a Higgs boson that is produced in association with a $W$ or $Z$ boson, has a mass in the range $90-150 \mathrm{GeV} / \mathrm{c}^{2}$, and decays into $b \bar{b}$ pair. We observe an excess of events in the data compared with the background predictions, which is most significant in the mass range between 115 and $135 \mathrm{GeV} / \mathrm{c}^{2}$, consistent with the Higgs-like particle recently observed by ATLAS and CMS. The most significant local excess is 2.7 standard deviations, corresponding to a global significance of 2.5 standard deviations. This result may provide the first evidence for the Higgs coupling to $b$ quarks and we are looking forward to the discovery of $H \rightarrow b \bar{b}$ at LHC.

\section{References}

[1] F. Englert and R. Brout, Phys. Rev. Lett. 13, 321 (1964); 

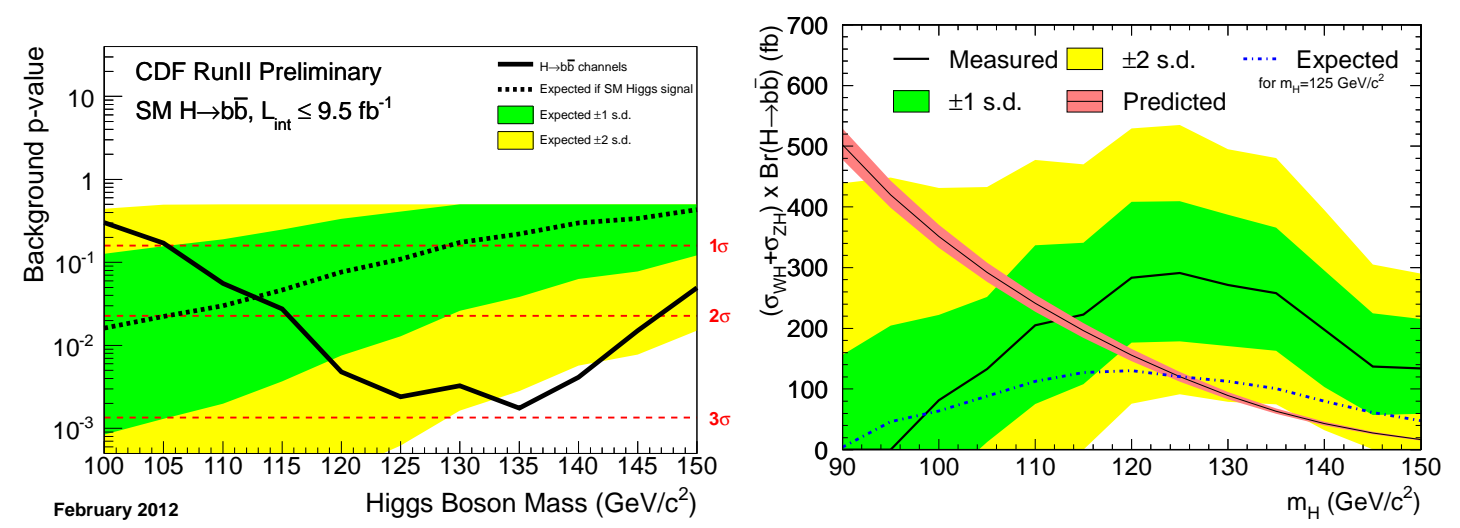

Figure 2: (Left) Background-only p-value for the combined search and the expected value assuming a SM Higgs signal is present as a function of $m_{H}$. (Right) The best-fit cross section times branching ratio as a function of $m_{H}$. The shaded regions show the one or two sigma bands and the SM prediction is shown as a smooth falling curve. The dot-dashes are what is expected for the SM Higgs signal of $m_{H}=125 \mathrm{GeV} / \mathrm{c}^{2}$.

P.W. Higgs, Phys. Rev. Lett. 13, 508 (1964);

G.S. Guralnik, C.R. Hagen, and T.W.B. Kibble, Phys. Rev. Lett. 13, 585 (1964).

[2] CDF and D0 Collaborations, Combined CDF and D0 Search for Standard Model Higgs Boson Production with up to $10.0 \mathrm{fb}^{-1}$ of Data, arXiv:1203.3774.

[3] CDF and D0 Collaborations, Combination of the top-quark mass measurements from the Tevatron collider, arXiv:1207.1069.

[4] CDF and D0 Collaborations, 2012 Update of the Combination of CDF and D0 Results for the Mass of the $W$ Boson, arXiv:1204.0042.

[5] The LEP Electroweak Working Group, Status of March 2012, http://lepewwg.web.cern.ch/LEPEWWG/.

[6] LEP Working Group for Higgs boson searches. (R. Barate et al.), Phys. Lett. B 565, 61 (2003).

[7] CMS Collab. (S. Chatrchyan et al.), Phys. Lett. B 710, 26 (2012).

[8] ATLAS Collab. (G. Aad et al.), Phys. Lett. B 710, 49 (2012).

[9] CMS Collab. (S. Chatrchyan et al.), Phys. Lett. B 716, 30 (2012).

[10] ATLAS Collab. (G. Aad et al.), Phys. Lett. B 716, 1 (2012).

[11] CDF Collab. (D. Acosta et al.), Phys. Rev. D 71, 032001 (2005).

[12] Tev4LHC Higgs Working Group, Standard Model Higgs cross sections at hadron colliders, http://maltoni.home.cern.ch/maltoni/TeV4LHC/SM.html.

[13] J. Freeman et al., Nucl. Instrum. Methods Phys. Res. A 697, 64 (2012).

[14] CDF Collab. (T. Aaltonen et al.), Phys. Rev. Lett. 109, 111804, (2012).

[15] CDF Collab. (T. Aaltonen et al.), Phys. Rev. Lett. 109, 111803, (2012).

[16] CDF Collab. (T. Aaltonen et al.), Phys. Rev. Lett. 109, 111805, (2012).

[17] CDF Collab. (T. Aaltonen et al.), Phys. Rev. Lett. 109, 111802, (2012). 